\title{
On the Growth and Form of Escherichia coli
}

\author{
By ARTHUR L. KOCH \\ Department of Biology, Indiana University, Bloomington, Indiana 47405, U.S.A.
}

(Received 1 December 1981)

\begin{abstract}
The shape of many micro-organisms can be understood in terms of the general surface stress hypothesis that hydrostatic pressure forces newly formed wall to expand in a particular direction. What distinguishes one type of organism from another is the regions of the cell where new wall growth occurs. For several classes of organisms, the pattern of growth deduced from the shape agrees with biochemical, morphological and physiological studies. Gram-negative rods, as typified by Escherichia coli, have a morphology that may be explained in several ways by this general hypothesis.

In the present paper, the morphological, autoradiographic and biochemical data concerning $E$. coli are reviewed. Thirteen models are considered; there is reason to reject most of them but one model that includes two others appears more satisfactory. All the models conform to the biophysical principles that it is impossible to turn over stress-bearing peptidoglycan without shape change and that growth of the sacculus requires the introduction of new oligosaccharides and their covalent linkage before cleavage of stress-bearing bonds. The model that best accounts for the experimental data assumes that, while addition of peptidoglycan all over the cell is possible and does occur, the effective surface tension is higher (and therefore the rate of wall growth is very small) in old poles than on the sides, and very much lower at the developing division sites (where the rate of wall growth is high). It is shown that, when the hydrostatic pressure is constant throughout the cell and during a large part of the cell cycle, changes in the biochemical mechanism of wall growth which correspond to a decrease in surface tension would lead to an invagination of the stress-bearing wall. An additional mechanism may, however, be needed for final closure and for the splitting of the last few covalent bonds holding the two nascent cells together.
\end{abstract}

\section{INTRODUCTION}

In several recent papers (Koch et al., 1981a, b, 1982; Koch, 1982), we have developed a general hypothesis to account for the morphology of most types of bacteria. The central concept is that in order to enlarge a closed vessel under pressure, new wall material must first be added and linked securely with multiple covalent bonds to the existing wall. The newly attached material is then necessarily in a relaxed, unextended configuration. In a second stage, certain bonds under tension in the pre-existing wall are selectively split so that the wall enlarges. As the new material comes to support the stress, it is stretched into an extended configuration due to the hydrostatic pressure. This determines the shape of the growing cell. In the present paper, I consider in detail a more complex case than treated previously, namely, Escherichia coli. This Gram-negative organism is not strictly rod-shaped; from inspection of many published electron micrographs, it does not have a region of constant diameter. There is evidence (Trueba \& Woldringh, 1980) that the shape and maximum girth vary throughout the cell division cycle. Moreover, the average width and length change depending on the nutritional environment of the cells (see, for example, Maaløe \& Kjeldgaard, 1966; Maaløe, 1978; Grover et al., 1978). Such changes are not seen with Gram-positive streptococci or Bacillus.

Any model of the development of the cell wall as it enlarges and divides must take into 
account the replication of the bacterial chromosome. The initiation of replication of the chromosome has figured prominently in theories of cell division (Pritchard, 1974; Lark, 1978; Helmstetter et al., 1979) since the Cooper-Helmstetter model was proposed (Cooper \& Helmstetter, 1968). However, the size of the cells at chromosome initiation is as disperse as the size at division (Koch, 1977; Koppes et al., 1978), and the evidence is now convincing that, within the same culture, chromosome replication in some cells may start early in the cell cycle while in others it may start late in the previous cell cycle. However, chromosome segregation and the existence of dichotomous replication still pose constraints on any proposed model.

The envelope of $E$. coli is now well understood. The peptidoglycan layer appears to be approximately one-third of a layer thick (Braun, 1975). This network appears to be the main stress-bearing element, but both the inner and outer membrane are impermeable to passage of proteins, except for special (usually biosynthetic) processes. The inner membrane is also generally impermeable to small molecules and inorganic ions. These membranes, while not mechanically strong, develop the osmotic pressure and transfer it to the peptidoglycan layer by virtue of being forced against it (inner membrane) or being attached to it by lipoprotein (outer membrane).

The essential features of the external morphology of this organism can be gleaned from countless electron micrographs that have been published in the last 30 years: $E$. coli has cylindrical symmetry with the axis having usually little or no curvature. The surface exhibits no discontinuities corresponding to wall bands such as are observed with Streptococcus faecium (Higgins \& Shockman, 1976) and Bacillus subtilis (Burdett \& Higgins, 1978). Of special importance is that the surface is continuous (i.e. there are no abrupt changes in slope) where the nearly cylindrical side-wall region meets the completed or nascent poles. The division furrow or constriction evident at cell division is the only discontinuity visible on the surface. Sometimes in sectioned material the developing wall is seen to invade the cytoplasm. This aspect has been most critically studied by Burdett \& Murray $(1974 a, b)$ and described as the external aspect of the ingrowing 'septal' structure. This is, however, unlike the septum of Gram-positive organisms, which consists of a thick cross-wall that is later split. When seen, the forming septal structure in $E$. coli consists of a cylindrically symmetrical invagination of both the inner membrane and peptidoglycan, both being continuous with the same structures in the rest of the wall. Consequently, there is a folded double layer of inner membrane and peptidoglycan. The outer membrane appears to cover, but not enter, the septal region and seems to prevent the septal area from pulling apart. Burdett \& Murray $(1974 a, b)$ suggest this may occur via lipoprotein (which is not found in the septal region) that may serve as a linker through the outer membrane holding the peptidoglycan leaflets together. Finally, there appears to be a 'gap' (electron transparent material) between the two planar surfaces of peptidoglycan. Burdett \& Murray suggest other factors may play a role in causing the peptidoglycan layers to adhere to each other, namely, direct hydrogen bonding or bonding via a hypothetical electron-transparent gap-substance. The key point, whether only the constriction furrow or further invagination is observed in the electron microscope, is that the peptidoglycan invaginates during the division process leaving the wall continuous, and a splitting process is not needed as it is for Grampositive bacteria.

In the presence of antibiotics that block wall formation, bulges form at septal sites (Schwarz et al., 1969; Hoffman et al., 1972; Burdett \& Murray, 1974a,b) as if the division furrow material evaginated.

The mode of wall growth of E. coli is dispersive (Van Tubergen \& Setlow, 1961; Lin et al., 1971; Schwarz et al., 1975). The experiments of Lin et al. (1971) showed that when cells with uniformly labelled walls were allowed to grow in methocellulose in the absence of exogenous labels, label was not lost or turned over but simply diluted by new growth. After three or four divisions, all portions of the short chains resolvable by light microscopy were uniformly radioactive. Ryter et al. (1973), Schwarz et al. (1975) and Verwer \& Nanninga (1980) have shown central zones of incorporation. De Pedro \& Schwarz (1981) have recently obtained biochemical results that are consistent with peptidoglycan being added without extensive cross-linking and slowly becoming more highly cross-linked into the covalent fabric of the wall. Schwarz (personal 
communication) suggests that initially new material is incorporated into the central zones and later becomes more peripherally distributed. For considerations of shape, only the step where stress-bearing bands are split is relevant. Consequently, the initial local accumulation of label should not be regarded as definite evidence for zonal growth of the stress-bearing wall.

While I consider the autoradiographic data demonstrating dispersive growth to be definitive, most proposed models of the cell cycle in $E$. coli are inconsistent with dispersive growth and have involved zonal growth either as semiconservative symmetrical or as asymmetrical growth. In this tradition, I, too, include narrow zonal processes amongst the possibilities as well as diffuse growth processes, and return to this question in the discussion.

\section{ZONAL GROWTH MODELS}

In the present paper, 13 models are considered (see Table 1). They fall into four classes: zonal, diffuse, variable $T$, and mechanisms with multiple growth modes. Models I and II are zonal models and are simple variants of the model we have proposed for $S$. faecium (Koch et al., $1981 a$ ). Both assume that all growth of wall takes place in a narrow growth zone. In both zonal models, growth occurs by two processes: one is the inward development of the septum, the other is the peripheral splitting of that septum with the addition of a little more wall material. The shape is determined by the angle of growth that allows the pressure-volume work due to new protoplasmic material to equal the surface tension-area expansion work needed to enlarge the wall. The two models differ only in the presumed trigger than initiates new growth zones.

For Model I, it is assumed that a new growth zone is initiated at the instant that the old one is completed. This zonal model can generate a variety of shapes depending on $P$, the hydrostatic pressure, $T$, the surface tension in the growth zone, $a$, the radius of the wall band as it splits to form two new wall bands and initiate the septum formation that defines the growth zone, and $\delta$, the thickness of the septum relative to that of the outer wall. The best fit for $S$. faecium was

\section{Table 1. Symmetric models for the form of Gram-negative rods}

The 13 models considered are listed ( $P$ is the hydrostatic pressure, $a$ is the radius of the pole and $T$ is the surface tension - see text). The exclusion of a model by the autoradiographic experiments of Lin et al. (1971) or by all other considerations (see Discussion) is indicated by Yes in the appropriate column.

$\begin{array}{cc}\overbrace{\text { Autoradiography }}^{\text {Model excluded by: }} \\ \text { Other } \\ \text { Yes } & \text { Yes } \\ ? & \text { Yes } \\ \bullet & \bullet \\ & \\ \text { Yes } & \bullet \\ \text { Yes } & \bullet \\ \text { Yes } & \text { Yes } \\ \text { Yes } & \text { Yes } \\ \text { Yes } & \bullet \\ \text { Yes } & \text { Yes } \\ \text { Yes } & \text { Yes } \\ \bullet & \bullet\end{array}$

Narrow Zonal Wall Growth

I. New growth zone initiated at division

II. New growth zone initiated at initiation of chromosome replication

III. Diffuse rectification of angular discontinuities

Diffuse Growth Over Entire Surface

IV. $T$ decreases in developing constriction sites

Poles and Sidewalls Formed Differently

V. Septum made and then stretched

a. cylindrical zonal sides: $P a / T=2$

b. cylindrical diffuse sides: $P a / T=1$

c. cylindrical diffuse sides: $P a / T>1$

VI. Zonal poles

a. cylindrical zonal sides: $P a / T=2$

b. cylindrical diffuse sides: $P a / T=1$

c. cylindrical diffuse sides: $P a / T>1$

VII. Diffuse poles

a. cylindrical zonal sides: $P a / T=2$

b. cylindrical diffuse sides: $P a / T=1$

c. cylindrical diffuse sides: $P a / T>1$ 

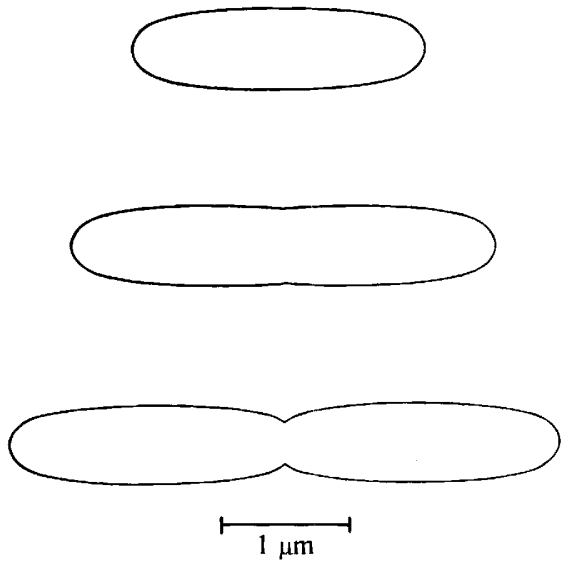

Fig. 1
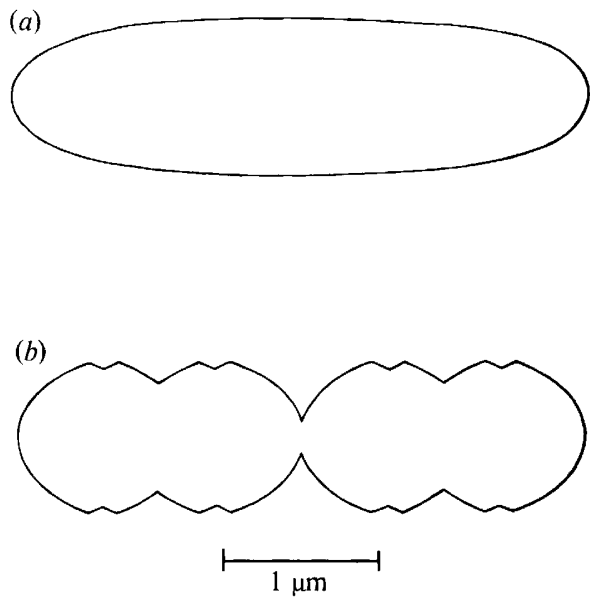

Fig. 2

Fig. 1. Shape of slowly growing E. coli, predicted by a narrow growth zone model (Model I of Table 1). This model assumes that wall growth occurs only at the septal region and that a new division site arises at completion of the previous division. The cell shape is calculated as indicated in the text to fit a real set of experimental data.

Fig. 2. Shape of fast growing E. coli (doubling time $24 \mathrm{~min}$ ), predicted by Model I ( $a$ ) and Model II $(b)$ of Table 1 (for details, see text). The latter model assumes that growth zones are initiated when chromosomal repication is initiated.

achieved (Koch et al., 1981a) by assuming that these quantities are all constant, that $P a / T$ is slightly less than 2 and that $\delta$ is 2 , at least as cell division is completed to form two daughter cells. It will be assumed here that $\delta=2$ for $E$. coli throughout the development of the pole.

The formula that describes the shape of the pole, when $\delta=2$, can be restated from equation (4) of Koch et al. (1981a) as follows:

$$
z=-(2 T / P) \ln \left\{1-(\operatorname{Pr} / 2 T)^{2}\right\}
$$

where $z$ is the axial distance from the tip of the pole to the locus where the radius is $r$. From measurements of the maximum radius and half length of a cell that has just completed division (thereby simultaneously specifying $z$ and $r$ ), the value of $P / T$ which satisfies equation (1) can be calculated. From this value of $P / T$, the entire shape of a pole within the confines of the zonal model (with $\delta=2$ ) can be generated. Such a fit is shown in Fig. 1 for a slowly growing culture of E. coli $\mathrm{B} / \mathrm{rA}$ ATCC 12407 with the dimensions taken from analysis of the data of Woldringh et al. (1977) and Trueba \& Woldringh (1980). Their measurements of cells showing constriction have been used to calculate the dimensions of the average newborn cell by the methods presented elsewhere (Koch \& Higgins, 1982).

Comparison of this calculated shape with the electron microscopic profiles shows good agreement, but perhaps the calculated shapes of the poles are not blunt enough. However, the discontinuity at the middle of the cell is infinitesimal - much too small to be detected experimentally. Also shown in Fig. 1 are calculated intermediary stages in the growth cycle. Throughout, the shape is quite consistent with photomicrographs. Figure $2(a)$ shows the calculated shape for Model I of a newborn cell of the same strain, but from a hypothetical culture with a doubling time of only $24 \mathrm{~min}$. As before, the calculations are based on the measurements from Woldringh's laboratory.

These calculations have been based on the assumption that a new growth zone is created at the instant of cell division. While it is reasonable to assume that DNA synthesis occupies only a portion of the total cell cycle for slowly growing organisms, for rapidly growing cells, chromosome replication takes longer than a cell doubling time. A model more consistent with 
our knowledge of the DNA replication cycle is Model II (Fig. $2 b$ ). The assumption that underlies this model is that growth zones are present for each replication site. An additional second assumption must be made to complete the specification of the cell shape, namely, that the completed pole volume is the same for fast and slow growing cells. This follows from the well known constancy of the ratio of protein to DNA of organisms in balanced cultures growing at different rates, and allows the pole height to be calculated from the measured maximal diameter. As has been clear since the original insights of Schaechter et al. (1962) and Cooper \& Helmstetter (1968), multiple replication forks must be present. Assuming that $C$, the time for DNA synthesis, lies between 40 and $45 \mathrm{~min}$ and that $D$, the gap before cell division, lies between 20 and $25 \mathrm{~min}$, then there would be three generations of chromosomes replication forks present in a newborn cell with a doubling time of $24 \mathrm{~min}$. The structure presented in Fig. 2(b) is consistent with these considerations. However, from the magnitude of discontinuities in the cell outline, Model II fails completely for the case of rapidly growing $E$. coli. Therefore, neither Model I nor Model II can be reconciled with the observed shape.

\section{DIFFUSE WALL GROWTH MODEL}

The alternative possible mode of growth is mathematically and conceptually much more complex. Models in this class assume that the enzymes and raw materials for wall extension are available over a large portion of the surface : the cell grows diffusely as a balloon or a soap bubble would grow when inflated. The models in this category (Models III, IV, Vb, Vc, VIb, VIc, VIIa, VIIb and VIIc of Table 1) hold that cells differ in two major ways from these inanimate objects. Firstly, each model assumes special processes that lead to cell division. Each model also assumes that wall growth takes place under constant pressure (as in the zonal models considered above) and that wall extension takes place only as the hydrostatic pressure tends to increase in response to protoplasmic synthesis and to the transport of new materials into the cell. [The pressure stays constant because a slight increase in pressure forces wall growth; this in turn causes an increase in the contained volume, which tends to lower the pressure. We have proposed a biophysical explanation of how the enzymes involved in cleavage of stress-bearing wall could serve to maintain the hydrostatic pressure (Koch et al., 1981 a).] When an ordinary spherical soap bubble is blown, the pressure is not constant. When more air is blown in, the bubble enlarges, but when equilibrium becomes re-established, the pressure will be less than it was originally. This is because bubbles obey the Young-Laplace law.

The Young-Laplace law (Boys, 1890; Thompson, 1942; Koch et al., 1982) is:

$$
P=T\left(1 / R_{1}+1 / R_{2}\right)
$$

where $R_{1}$ and $R_{2}$ are the principal radii of curvature of any point on a membrane surface. For a sphere of radius $a, R_{1}=R_{2}=a$, and $P=2 T / a$. From this it can be seen, as first shown experimentally by Plateau (1873), that the larger the radius, the smaller the pressure.

While a free-floating closed bubble must be spherical, with additional constraints many other shapes are possible. For a cylindrical bubble, one of the radii of curvature is infinite, and if the other radius is equal to $a$, then $P=T / a$. As with a spherical bubble, increasing the radius of the cylinder would lower the equilibrium pressure. In a cylindrical soap bubble with hemispherical caps, such that the radius of the cylinder and the caps were $a$, and in which $P a / T$ was 1 , the cylindrical part would be stable, but the caps would tend to expand (or contract) until their radius of curvature was doubled. Figure 3 shows the two possible pole shapes for rod-shaped organisms that obey the Young-Laplace law over their entire surface. Clearly, these are not similar in shape to any known micro-organism. Moreover, the shape would not be stable unless the material at the discontinuities (i.e. the end of the cylindrical region) was more rigid than the remainder.

\section{Computer simulations}

To consider the implications of the diffuse models further, we present computer-calculated shapes of cylindrical surfaces. These were computed from the numerical integration of the 


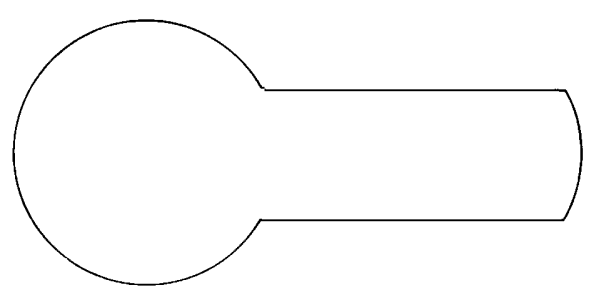

Fig. 3. Hypothetical rod whose surface grows uniformly all over. A soap bubble could be blown in this shape, since equation (2) (see text) shows that the pressure in equilibrium with the surface would be the same in all regions. It could only be blown if there were two rigid rings at the discontinuities. Obviously, a dumbell-shaped object or an object with two almost flattened ends could be formed. Both poles in the diagram have radii twice that of the central cylindrical part.

following equation where $r$ and $z$ are as defined above and $S$ is the slope, $\mathrm{d} r / \mathrm{d} z$ :

$$
S=\left[4 r^{2} /\left\{2 r_{0} /\left(1+S_{0}^{2}\right)^{1 / 2}-P\left(r_{0}^{2}-r^{2}\right) / T\right\}^{2}-1\right]^{1 / 2}
$$

The derivation of a different form of this equation has been presented previously (Koch et al., 1982). Given $r_{0}$ and $S_{0}$, the radius and slope respectively at a fixed point, the computer calculates the trajectory of the cylindrical surface. It does so by calculating a value of $S$ from equation (3) and then a new value of $r$ from $r=r_{0}+S \Delta$, where $\Delta$ is the incremental axial distance (usually $1 / 10000$ of the original radius). The computer repeats this cycle over and over again thousands of times. The surface that spans two parallel co-axial rings of specified radius, $a$ and $b$, and separation distance, $L$, must be calculated by trial and error. The calculation is unwieldy because small differences are often involved and for this reason the computer programs use different, but equivalent, forms of equation (3) to decrease rounding errors (see Koch et al., 1982). In addition, where needed, double precision arithmetic has been used ( 24 decimal places) so that the final results are correct to 5 decimal places. An additional difficulty in computation is the assignment of sign to the square roots. In the present version, the computer stops when a branch of the curve is completed; the necessary branches have been combined to form the continuous curves. The program written for a $720 \mathrm{C}$ Wang minicomputer was tested in a number of ways: it accurately generated known shapes such as the profiles of spheres, cylinders and catenoids when the parameters were suitably chosen. This program has been used for other purposes (Koch et al., 1982), mainly to define conditions under which diffuse growth can lead to cylinder elongation. Here it is used for additional purposes, including the study of cylindrical growth where the radius is not constant and of constrictive division. For the latter process, $T$ can no longer be taken to be constant, and the cases where $T$ is proportional to $r$ raised to various powers are considered.

\section{Side-wall growth with rigid poles}

In previous papers (Koch et al., $1981 a, b, 1982$ ), we considered mechanisms for side-wall growth that can generate cylinders of constant diameter. The narrow zonal elongation model requires that $P / T=2 / a$, while the diffuse elongation model requires that $P / T=1 / a$. Either of these modes of growth leads to a model for the cell diameter in which the biological conditions determining $P$ and $T$ secondarily fix the radius. Both models require additional mechanisms to keep the radius stable. The zonal model is not stable if the radius becomes too large; the diffuse model is not stable if the length becomes too long. In this section, I consider diffuse growth with $P / T$ slightly greater than $1 / a$ (Models Vc, VIc and VIIc of Table 1). This leads to progressive swelling as growth proceeds and to an acceptable model of growth only if the central swelling is secondarily compensated by a separate mechanism, which does so as part of the process of pole formation. Although the old poles grow little, since there is half the tension developed in their walls, eventually they would get wider. As pointed out before (Koch et al., 1982), this would tend to lead to fatter and more spherical cells. Below, further consideration of the cell poles will be given, but in this section the caps have been assumed to have a rigid shape. A computer 


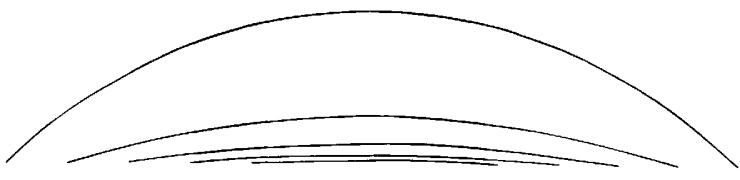

$2 a$

Fig. 4. Side-walls for Models Vc and VIc of Table 1. The curves were calculated for a diffuse model assuming that the poles are rigid and that $P a / T=1 \cdot 1$, i.e. $10 \%$ above the value that would give cylindrical elongation. A series of progressively longer side-wall shapes is shown, culminating with a spherical side-wall. Further growth would lead to length contraction as the maximum radius increased. However, it is assumed that some other cellular process initiates the next division as shown on the bottom of the figure. The diameter at the poles is indicated by the central bar.

simulation, using the facility described in the previous section, showed that elongation concomitant with swelling is possible (Fig. 4). The value of $P a / T$ was fixed at $1 \cdot 1$; this is $10 \%$ in excess of the value that is necessary to maintain a regular cylinder of radius $a$. Starting with a rod-shaped organism with rigid poles, diffuse uniform growth over the entire lateral surface, with $\mathrm{Pa} / \mathrm{T}$ slightly greater than 1 , leads to elongation with a progressively increasing degree of bulging. The profiles of the caps have been omitted from Fig. 4. As growth takes place, the caps are imagined to be simply pushed apart. The diameter of the pole is $2 a$ in all cases and is indicated by the central bar. The degree of bulging increases as lateral wall grows from a length of $50 \%$ to $150 \%$ of the diameter of the poles. Such a growth process cannot continue indefinitely and must be abrogated when the profile becomes circular. This is the point (the longest profile shown on the top of Fig. 4) where the surface becomes part of a spherical segment. Further volume increase, with the restriction that the terminal radius is $a$, leads to width expansion and length contraction and the formation of a disc-shaped object with the caps on the faces of the disc. Models Vc, VIc and VIIc of Table 1 assume that growth ordinarily does not lead to such shapes because of the normal processes leading to cell division. A possibility of a developing, central, ingrowing region is shown on the lower portion of Fig. 4. Obviously, the shapes attained by the more complex process of growth plus development of new cell poles depend on the cellular mechanism that triggers the formation of the new division site. The profile shown is the shape that results if a $10 \%$ increase in diameter triggers the site of a new division whose radius is fixed at $a$ by an unspecified process.

\section{Can poles be formed by diffusive growth with $P / T$ constant?}

Poles of Gram-positive cocci appear to divide by septation in two ways (Koch et al., 1982). First, as in the case of $S$. faecium (Higgins \& Shockman, 1976), a wall band splits and septal material forms between; the septum then progressively splits to form new poles. This is the case for which the zonal dome growth model was derived (Koch et al., 1981 a). The zonal dome model assumes that the only place that new external wall growth occurs is in a very narrow region near the splitting septum. The other class of more spherical organisms form cross-walls that when complete, or nearly complete, split. The more likely model for this case is that reworking and adding of new material takes place all over the split portion of the septum and results in a spherical structure. Both types involve the formation of a septum. Can cell division take place with no septum as a constrictive process by diffuse growth with $P$ and $T$ constant, as in the above two cases? The answer is no, but the simulations suggest that a zonal mechanism with a little additional diffuse character (Model III) might account for the shape of $E$. coli.

The question may be reformulated as follows. A cylindrical bubble can be drawn out so far that its circumference equals its length. Until it achieves that length a simple cylinder is the only 


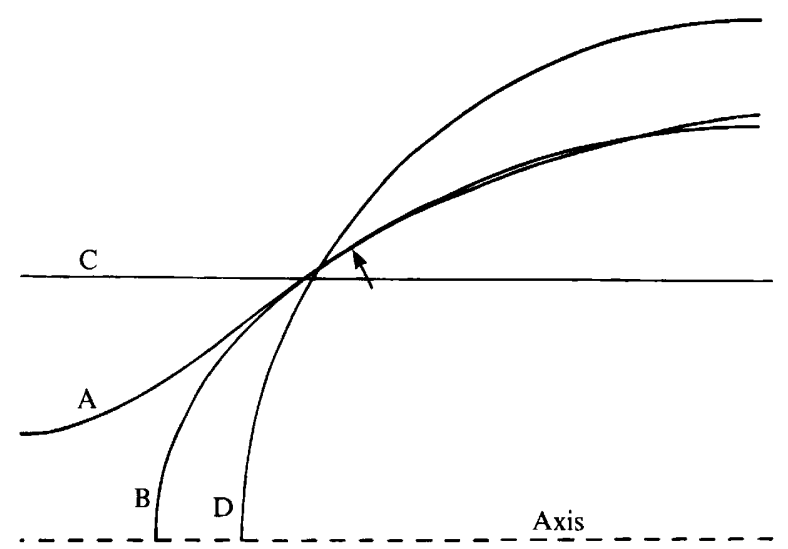

Fig. 5. Constrictive models of growth with $T$ constant. Curve A shows the profile of a portion of a hypothetical cell where uniform diffuse wall growth occurs. This curve satisfies the same value of $P a / T$ as does curve $\mathrm{C}$, corresponding to cylindrical elongation, or curve $\mathrm{D}$, corresponding to a spherical dome. Curve B corresponds to a zonal dome (Model I of Table 1) with exactly the same slope at the arrow. The similarity of curves A and B over much of their length suggests a model of intermediate character. This is Model III of Table 1 which postulates zonal growth and some diffuse growth (or turnover) which smoothes the angular discontinuities predicted by Model II (see Fig. 2) for fast growing cells to yield the smooth contours actually observed.

shape possible. However, above this critical length, a range of shapes is possible. For example, a soap bubble or a flowing jet of water forms constrictions and expansions. When by chance the radius becomes zero, separation of the cylinder into smaller bubbles or droplets occurs. Could this be the physical basis for cell division in some rod-shaped organisms? For the mechanism to work, the cells need to be long and instabilities need to arise to allow growth to cause the cell to bulge in some places and cause it to contract as it grows in others. The biological origin of the fluctuations in slope would have to be explained in some independent way, but the energy needed to give rise to the constriction would be readily understood as ultimately deriving from the expansion work available from macromolecular synthesis and transport of solutes into the cell.

Figure 5 shows a curve (curve A) calculated in an attempt to approximate the quartile profile of a constricting bacterium. It is well known (Thompson, 1942) that a solution that leads to zero radius corresponds to a sphere (curve $\mathrm{D}$ ). (This leads to a model for cell division in which $T$ varies so that $P a / T=2$ in the pole region and $P a / T=1$ in the cylindrical region, see below. But that is not the case under consideration in this section.) Two other profiles of surfaces of cylindrical symmetry are shown (curves $C$ and D). These are solutions for the same value of $P$ and $T$. Curve $C$ corresponds to a cylinder; curve $D$ to a sphere. While there are a family of curves for a given value of $P / T$, curve A was calculated to go through the point indicated by the arrow with the same slope as does curve B, calculated from equation (1) for a zonal dome model.

The parameters have been chosen so that the surfaces are exactly superimposed at the region indicated by the arrow. However, over a considerable region, these curves are quite similar. Consequently, Model III is proposed; this is similar to Model II but modified to permit some very limited further growth and reworking of the wall. This would lead to expansion with erasure of the protruding discontinuities. It can readily be shown that diffuse growth would have its most dramatic effect on regions with smaller radii of curvature. Therefore, limited diffuse wall growth would leave the pole essentially as calculated by the zonal model but blur the more central angular regions.

If, however, diffuse wall growth were to continue in an unlimited fashion, then the surface structure would become the spherical curve $\mathrm{D}$ because it has been assumed that $P / T$ is constant all over the surface of the cell, and if maintained, the sphere would have the dimensions indicated. 


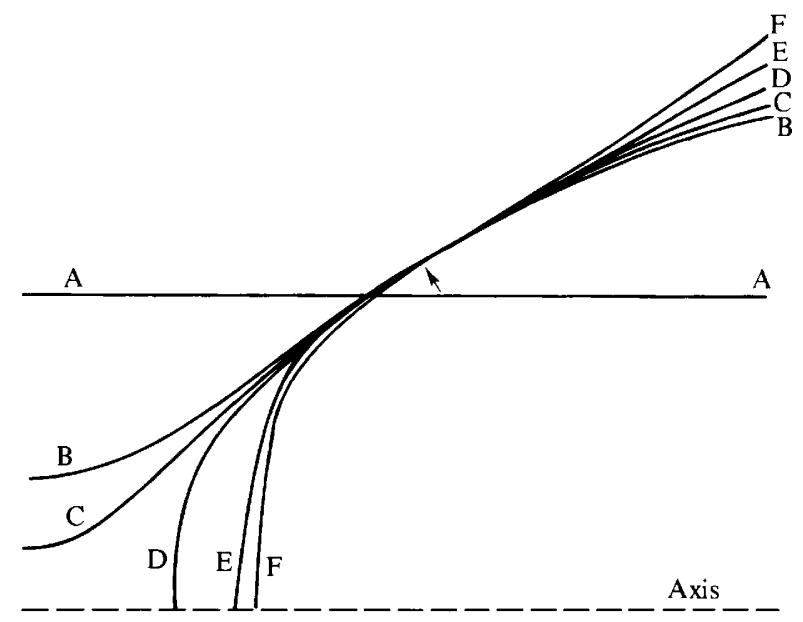

Fig. 6. Variable $T$ model. Profiles for cells are shown where $T$ is a function of $r$. In A and B, $T$ is independent of $r$; in C, $T$ is proportional to $r^{1 / 2}$; in $\mathrm{D}, T$ is proportional to $r$; in $\mathrm{E}, T$ is proportional to $r^{2}$; and in $\mathrm{F}, T$ is proportional to $r^{3}$. All curves pass through the point marked by the arrow with the same slope. Only when $T$ is proportional to $r$ to the power of one or greater can closure (cell division) be achieved.

\section{Variable $T$}

If a glass tube is heated over a small section, surface tension causes constriction and can lead to the separation of the tube into two. This leads to a different kind of model, designated Model IV (Table 1). This is a model in which the value of $T$ decreases in the central part of the cell leading to invagination of newly added wall. To calculate such shapes, the computer program has been modified so that $T$ can be altered as the numerical projection of the surface continues. If $T$ becomes a function of $r$, and approaches zero as $r$ approaches zero, then completion of division is possible (Fig. 6). However, it can be shown from equation (3) (and demonstrated with the computer) that not all possible ways for $T$ to approach zero as $r$ approaches zero lead to closure. If $T$ is proportional to $r$ raised to a power less than one, the slope becomes zero before $r$ reaches zero. In this case, a constriction, but not a constriction with discontinuity, would be produced and no division could occur. To obtain conditions that lead to closure of a growing, constrictive form, the dependency of $T$ on $r$ and the slope at a particular part must be chosen consonantly. Figure 6 shows the shapes for curves passing through the point designated by the arrow. This point and the shape of the curves at the point are the same as in Fig. 5. These curves were calculated for a series of different relationships; i.e. where $T$ is constant (curves $A$ and $B$ ), or is proportional to $r^{1 / 2}$ (curve C), to $r$ (curve D), to $r^{2}$ (curve E), or to $r^{3}$ (curve F).

So far, I have taken $T$ as a parameter that may be altered by the cell. It is by definition the amount of work required to enlarge the surface by a unit increment in area. Discussion of the possible ways that the cell might alter $T$ will be presented elsewhere. Here it will suffice to give one example of how the cell might decrease $T$ from one time and place to another. In high $T$ regions, single glycan chains might be inserted, whereas in low $T$ regions, groups of precrossbridged glycan would be inserted. If this process actually takes place, $T$ might become smaller but could never become zero. Thus, even if the capacity for wall enlargement is very large in a central zone, very rapid invagination of the wall is possible, but may not lead to the closure that is needed for cell division. If equation (2) holds, then the constricted regions will satisfy $r=T / P$ (see Fig. 7). Consequently, if the value of $T$ in the growth zone is one-tenth that in the cylindrical part, the radius of the most invaginated part will be one-tenth that of the cylindrical part. Therefore, for closure to be effected, some secondary mechanism is needed. The final closure could be mediated by protein(s) that can exist in multimeric units and span the remaining gap, bind the peptidoglycan and cleave the units holding the two future cells together. This may be an ideal role for some minor penicillin-binding protein. 


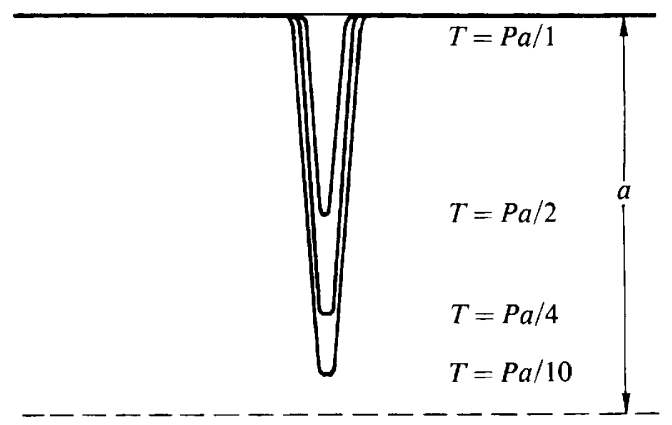

Fig. 7. Model IV of Table 1, in which a narrow region has a progressively higher $T$ as constriction proceeds. The curve shows diagrammatically the progressive invagination as the narrow zone achieves a smaller and smaller value of $T$. Only for $T=0$ would closure occur. Therefore some additional mechanism is needed for final closure.

\section{MODELS IN WHICH SIDE-WALLS AND POLES ARE GENERATED DIFFERENTLY}

Models I and II operate by a single mechanism to produce both the poles and side-wall. Models III and IV assume variation between these regions of the cell. In this section, models are presented that assume a different but simple mechanism to function in the formation of the poles and side-wall. In Table 1, categories for the side-walls of a zonal and two diffuse models and for the poles of a mechanical, a zonal, and a diffuse model are indicated (Models V, VI and VII). Relatively little discussion of the nine combinations is needed since most of these have been considered previously (Koch et al., 1981 a, 1982) or have been discussed above.

For side-walls of constant diameter, $\mathrm{Pa} / \mathrm{T}$ must be exactly 2 for the zonal, and exactly 1 for the diffuse model, and require non-trivial cellular mechanisms to maintain the appropriate values of $P$ and $T$. The second diffuse model for sides with $P a / T$ slightly greater than 1 leads to slightly bowed outside walls (see above). While not requiring control on $\mathrm{Pa} / \mathrm{T}$, it requires some other mechanism to establish the centre of the cell and fix the radius of newly formed poles. Zonal dome growth could yield variously shaped poles, but uniform diffuse growth yields only hemispherical shapes. There is one additional possible class of mechanisms yielding a cell pole. This is the mechanical deformation of a split septum.

\section{Pole shape determined by pressure on the split septum (Model V)}

The model considered in this section is quite different from the zonal or diffuse models for pole formation and could apply to the poles of Gram-positive organisms. In the version for enteric organisms, it assumes that a double peptidoglycan layer is formed through a diameter of the cell so that on completion the poles are flatter than old poles since this invaginating portion of the wall is not subject to the stress experienced by the outside wall to the degree that the outer membrane shares the stress over this small region. Consequently, its formation would be different from that of the cylinder wall. While the peptidoglycan can be linked, as in external wall formation, the cutting of existing cross-links as it grows inward would be subject to different rules, as there is little tension in the developing septum to guide its development (Koch et al., $1981 a$ ). If full tension is not present after the cleavage step then, when the cell division is complete, each half would have the properties of a sheet of some stretchable material (e.g. a sheet of rubber). Consequently, the new poles of both daughter cells could expand as the synthesis of protoplasm continues. When stretched, it would approximate a parabola, or a catenary of revolution, or some other more complex shape. The shape would be parabolic if the original peptidoglycan layer was uniformly stretchable all over the surface. Other shapes would be generated if the physical properties differed with distance from the axis of symmetry. 


\section{Location of the centre of the side-walls}

Asymmetric growth can solve the problem of localizing the new central site that fixes the position of the next cell division. Various asymmetric models have been catalogued by DaneoMoore \& Shockman (1977) and Sargent (1979). They will not be considered further since for $E$. coli they have been critically excluded by the autoradiographic studies of Verwer \& Nanninga (1980); but the problem of location of the centre remains.

Models IV, V, VI and VII of Table 1 require that a special mechanism exists to locate and to create de novo a new site for cell division at which the development of two new poles will be initiated. For the other types of mechanism considered here, and for the growth of the streptococci ; the division sites arise from previous division sites. This problem and a Renovated Replicon Model to fix the division site accurately in the centre of a Gram-positive rod have been discussed (Koch et al., 1981 b). Because there appears to be no evidence for any structure or even the existence of a junction between the pole and side-wall of $E$. coli, it is suggested that the only two distal and unique sites within a Gram-negative rod would be at the very tips of the poles. If attachment of unique proteins and origin or terminus DNA to these two points in the cell could be shown, then the obvious extension of our Renovated Replicon Model to $E$. coli would be very attractive. Such unique points in the cell would be a natural consequence of Model IV and at least not prohibited by other models.

\section{DISCUSSION}

In an earlier paper (Koch et al., 1981a), the biophysical aspects of the biosynthesis and degradation of chemical bonds under mechanical stress were discussed. For the pressures and thicknesses of walls in bacteria, considerable tension exists on the bonds of the peptidoglycan bearing the stress. This makes it easier for the bonds to be split by hydrolysis. For the case of $E$. coli, it was calculated that the negative free energy of hydrolysis of closely packed glycan chains would increase by $30 \%$. The increase would be several-fold larger for splitting the peptide chains, even if all possible cross-bridges were present and shared the stress evenly. The actual increase should be larger still, particularly where there are gaps or spaces in the network. The difference in energy, although only approximate, is about that of a high-energy phosphate bond. It consequently would be impossible to remove and replace a unit in the wall that is bearing the stress since there would be insufficient free energy in the transpeptidation process; therefore extensive turnover could not occur. If a region of peptidoglycan were removed at random and were replaced by a larger number of unextended glycan units, the wall would enlarge and eventually the cell would become spherical.

The 13 symmetrical models considered (Table 1) can be reduced considerably. Model I can be excluded, if it is assumed that there is a connection between DNA initiation and cell division. Model II can be excluded because of the absence of angular discontinuities in rapidly growing cells. However, the modified zonal model III and those models in which the poles and side-walls are formed differently cannot be so excluded. For diffuse side-wall formation where $P a / T=1$, only rigid poles are possible, thus excluding VIIb, while $\mathrm{Vb}$ and $\mathrm{VIb}$ remain as possibilities. Since loss of peptidoglycan does not take place in $E$. coli (see Introduction) and the radioactivity of all portions of the cell wall is diluted during a chase, only Models IV and VIIc and, possibly, III can be retained. These three may not be much different and further theoretical work will be devoted towards elaborating these models and calculating patterns of dilution of wall label.

(It may be noted that model VIIa includes a case for hemispherical poles such that $P a / T=2$ would apply both to poles and side walls. It may apply to some other organism.)

It is hard to fault the published studies on cell morphology, autoradiographic experiments, and the relationship of chromosome replication to cell division. At present, the most crucial experiment is to establish whether old poles incorporate any new peptidoglycan. A second, complementary experiment is to label a culture in the steady state, chase for at least 10 generations, and then search with electron-microscope high resolution autoradiography for the rare labelled poles. The degree of conservation will delimit these models. Both Models IV and VIIc would predict some, but not necessarily complete, conservation of the pole label. 
From the discussion presented above, there must be a metabolic difference between a forming pole, the side-wall region and old cell pole. Models III, IV, VIIb and VIIc all require that wall enlargement activity be largest in the developing pole, decreasing through the side-wall to the old pole. All of these possibilities are included within Model IV. Models VIIb and VIIc predict a morphological and possible biochemical discontinuity between poles and side-wall regions. While morphological discontinuities have not been seen, it is possible that new fixation techniques will reveal them. It is probable that the genes for penicillin-binding proteins will be cloned; antibodies to their products thus made available may enable the location of the proteins in different parts of the cells to be determined.

In summary, it is not necessary yet to postulate contractile forces, such as those acting through the cytoskeletons of eukaryote cells, to cause cell division in E. coli. Rather it appears that some variant of a variable $T$ model functions and the cell alters the energetics of external wall formation in order to achieve constriction and division.

Both the work and the title were inspired by the works of D'Arcy Wentworth Thompson and Robert Pritchard. The careful and painstaking studies of the electron microscopists Ian Burdett, Michael Higgins, Nanne Nanninga, Conrad Woldringh and their colleagues are of critical importance in the development of these ideas as were discussions with them. As in the first paper in this series, the ideas finally gelled at the Gordon conference on the bacterial cell wall, and I thank Elio Schaechter and Maxime Schwartz for permitting me to attend. Work in my laboratory is supported by the National Science Foundation under Grant PCM 79-11241.

\section{REFERENCES}

Boys, C. V. (1890). Soap Bubbles and the Forces which Mould Them. Society for the Promotion of Christian Knowledge, London. Reprinted by Dover: New York (1959).

BRAUN, V. (1975). Covalent lipoprotein from the outer membrane of Escherichia coli. Biochimica et biophysica acta 415, 335-377.

Burdett, I. D. J. \& Higgins, M. L. (1978). Studies of pole assembly in Bacillus subtilis by computer reconstructions of septal growth zones seen in central, longitudinal, thin sections of cells. Journal of Bacteriology 133, 959-971.

Burdett, I. D. J. \& Murray, R. G. E. (1974a). Septum formation in Escherichia coli: characterization of septal structures and the effect of antibiotics on cell division. Journal of Bacteriology 119, 303-324.

BurdetT, I. D. J. \& Murray, R. G. E. (1974b). Electron microscope studies of septum formation in Escherichia coli strains $\mathrm{B}$ and $\mathrm{B} / \mathrm{r}$ during synchronous growth. Journal of Bacteriology 119, 1039-1056

Cooper, S. \& Helmstetter, C. E. (1968). Chromosome replication and division cycle of Escherichia coli. Journal of Molecular Biology 31, 519-540.

Daneo-Moore, L. \& Shockman, G. D. (1977). The bacterial cell surface in growth and division. In The Synthesis, Assembly and Turnover of Cell Surface Components, pp. 597-715. Edited by G. Poste \& G. L. Nicolson. Amsterdam: Elsevier/North Holland.

De Pedro, M. A. \& Schwarz, U. (1981). Heterogeneity of newly inserted and pre-existing murein in the sacculus of E. coli. Proceedings of the National Academy of Sciences of the United States of America 78, 5856-5860.

Grover, N. B., Woldringh, C. L., Zaritsky, A. \& Rosenberger, R. F. (1978). Elongation of rodshaped bacteria. Journal of Theoretical Biology 67, 181-193.
Helmstetter, C. E., Pierucci, O., Weinberger, M., Holmes, M. \& TANG, M. S. (1979). Control of cell division in Escherichia coli. In The Bacteria Vol. VII, pp. 517-579. Edited by I. C. Gunsalus \& R. Y. Stanier. New York: Academic Press.

Higgins, M. L. \& Shockman, G. D. (1976). Study of a cycle of cell wall assembly in Streptococcus faecalis by three-dimensional reconstructions of thin section of cells. Journal of Bacteriology 127, 1346-1358.

Hoffman, B., Messer, W. \& Schwarz, U. (1972). Regulation of polar cap formation in the life cycle of Escherichia coli. Journal of Supramolecular Structure 1, 29-37.

KoCH, A. L. (1977). Does the initiation of chromosome replication regulate cell division? Advances in Microbial Physiology 16, 49-98.

KoCH, A. L. (1982). The shape of the hyphal tips of fungi. Journal of General Microbiology 128, 947-951.

Koch, A. L. \& Higgins, M. L. (1982). Cell cycle dynamics inferred from the static properties of cells in balanced growth. Journal of General Microbiology (in the Press).

KoCH, A. L., Higgins, M. L. \& Doyle, R. J. (1981 a). Surface tension-like forces determine bacterial shapes: Streptococcus faecium. Journal of General Microbiology 123, 151-161.

Koch, A. L., Mobley, H. L. T., Doyle, R. J. \& StreIPS, U. N. (1981 $b$ ). The coupling of wall growth and chromosome replication in Gram positive rods. FEMS Microbiology Letters 12, 201-208.

Koch, A. L., Higgins, M. L. \& Doyle, R. J. (1982). The role of surface stress in the morphology of microbes. Journal of General Microbiology 128, 927945.

Koppes, L. J. H., Woldringh, C. L. \& Nanninga, N. (1978). Size variation and correlation of different cell cycle events in slow-growing Escherichia coli. Journal of Bacteriology 134, 423-433. 
LARK, K. G. (1978). Some aspects of the regulation of DNA replication in Escherichia coli. In Biological Control and Development, pp. 201-217. Edited by R. F. Goldberger. New York: Plenum Press.

Lin, E. C. C., Hirota, Y. \& Jacob, F. (1971). On the process of cellular division in Escherichia coli. VI. Use of a Methocel-autoradiographic method for the study of cellular division in Escherichia coli. Journal of Bacteriology 108, 375-385.

MAaløE, O. (1978). Regulation of protein synthesizing machinery-ribosomes, rRNA, factors, etc. In Biological Control and Development, pp. 487-542. Edited by R. F. Goldberger. New York: Plenum Press.

MaAløe, O. \& KJeldgaARd, N. O. (1966). Control of Macromolecular Synthesis. New York: W. A. Benjamin.

Plateau, J. A. F. (1873). Statique Expérimentale et Théorique des Liquides Soumis aux Seules Forces Moléculaire. Paris: Gauthier-Villars.

PrITCHARD, R. H. (1974). On the growth and form of a bacterial cell. Philosophical Transactions of the Royal Society B267, 303-336.

Ryter, A., Hirota, Y.\& Schwarz, U. (1973). Process of cellular division in Escherichia coli. Growth pattern of E. coli murein. Journal of Molecular Biology 78, 185-195.

SARGENT, M. (1979). Surface extension and the cell cycle in prokaryotes. Advances in Microbial Physio$\log y$ 18, 105-176.

Schaechter, M., Williamson, J. P., HoOD, J. R., JR \&
Kосн, A. L. (1962). Growth, cell and nuclear divisions in some bacteria. Journal of General Microbiology 29, 421-434.

Schwarz, U., Asmus, A. \& Frank, H. (1969). Autolytic enzymes and cell division of Escherichia coli. Journal of Molecular Biology 41, 419-429.

Schwarz, U., Ryter, A., Rambach, A., Hellio, R. \& Hirota, Y. (1975). Process of cellular division in Escherichia coli. Differentiation of growth zones in the sacculus. Journal of Molecular Biology 98, 749759.

ThOMPSON, D'ARCY, W. (1942). On Growth and Form, 2nd edn, pp. 351-384. Cambridge: Cambridge University Press.

Trueba, F. J. \& Woldringh, C. L. (1980). Changes in cell diameter during the division cycle of Escherichia coli. Journal of Bacteriology 142, 869-878.

Van Tubergen, R. P. \& Setlow, R. B. (1961). Quantitative radioautographic studies of exponentially growing cultures of Escherichia coli. Biophysical Journal 1, 589-625.

VERWER, R. W. H. \& NANNINGA, N. (1980). Pattern of meso-DL-2,6-diaminopimelic acid incorporation during the division cycle of Escherichia coli. Journal of Bacteriology 144, 327-336.

Woldringh, C. L., De Jong, M. A., VAN Den BerG, W. \& KopPES, L. (1977). Morphological analysis of the division cycle of two Escherichia coli substrains during slow growth. Journal of Bacteriology 131, 270 279. 